\title{
Normal muscle strength and fatigability in patients with effort syndromes
}

\author{
Maria J Stokes, Robert G Cooper, Richard H T Edwards
}

\begin{abstract}
To examine fatigue mechanisms in an unselected series of patients with excess fatigue ("effort syndromes") their muscle function was compared with that of normal subjects. Voluntary performance was assessed with a cycle ergometer to exhaustion and by maximal isometric contractions of the quadriceps femoris. The mean maximal heart rate in patients during ergometry was $89 \%$ of the predicted rate, and quadriceps strength was either normal or was inappropriate for the available muscle, which suggested submaximal effort. Contractile performance was examined in the absence of volition with stimulated contractions of the adductor pollicis. During stimulated fatiguing activity patients were neither weaker nor more fatigable than controls; thus the excess fatigue experienced by the patients was not due to a defect of the contractile apparatus. The increased perception of effort must therefore be due to impairment of central rather than peripheral mechanisms.
\end{abstract}

The optimal approach to treatment of effort syndromes combines physical and psychological techniques.

\section{Introduction}

"Effort syndrome" is an ill defined diagnostic term embracing various disorders including breathlessness and effort intolerance, ' $\mathrm{Da}$ Costa's syndrome, ${ }^{2}$ vasoregulatory asthenia and neurasthenia, ${ }^{34}$ and postviral fatigue syndromes, ${ }^{5}$ including myalgic encephalomyelitis $^{6}$ (Royal Free disease ${ }^{7}$ ). There is no reliable, objective method for confirming the diagnosis, which must therefore rely on clinical criteria alone. The most common symptoms are weakness and excessive fatigue, which are often worse after exercise. ${ }^{+}$Some patients also complain of lassitude, lack of mental drive and concentration, muscle pain, and an intermittent feeling of general ill health that can be so incapacitating that everyday activities cannot be performed. Owing to their poor cardiovascular fitness patients may also experience during everyday activities symptoms (such as tachycardia) usually associated with intense exercise. ${ }^{3}$ Some patients show evidence of chronic infection with enterovirus (such as Coxsackie B), but even some patients reported as having the postviral fatigue syndrome have the symptoms without having had a viral infection. ${ }^{8}$ In one study most patients with postviral fatigue showed abnormal electrophysiological activity which seemed to indicate disturbed excitation of the sarcolemmal membrane. ${ }^{9}$ This observation, however, cannot entirely be explained by the presence of a virus as the same abnormality occurs with atrophy caused by disuse.'

A recent review of the postviral fatigue syndrome highlighted the flaws in previous studies - namely, the failure to define and study fatigue accurately, poor assessment of psychological features, and the use of inappropriate control groups. ${ }^{10}$ Fatigue is defined as failure to maintain a required force or output of power during sustained or repeated muscle contractions, and its causes are classified as being central or peripheral. ${ }^{.1}$ Central fatigue is due to failure of neural drive; it may result from lack of motivation, from impaired firing frequency at or proximal to the anterior horn cell, or from impaired recruitment of motor units. Peripheral fatigue results from failure at or beyond the neuromuscular junction and can be shown during stimulated contractions.

This study examined fatigue mechanisms in patients with effort syndromes by using well established techniques that are in regular clinical use. ${ }^{11 \cdot 14}$ Central fatigue was examined by testing the exercise capacity of the whole body and by testing the strength of the quadriceps femoris on voluntary movement, and peripheral fatigue was examined by using stimulated activity of the adductor pollicis to test strength and fatigability in the absence of volitional influences.

\section{Subjects and methods}

Thirty patients aged $21-50$ (mean 36.5 years; 18 women, 12 men) and 20 normal subjects aged $22-50$ (mean 30.5 years; 7 women and 13 men) were studied. The patients had a history of excessive general and muscular fatigue over one to 19 years but no evidence of neuromuscular disease on clinical, biochemical, histological, or histochemical examinations. Their functional capacity was hampered severely, to the extent that their lives were greatly disrupted. Not all patients had a history of viral infection related to the onset of symptoms. Informed consent was obtained from all participants, and the investigation was approved by the Liverpool Area Health Authority ethics committee.

A progressive resistance exercise test to exhaustion was performed in 15 patients (eight men and seven women) with a cycle ergometer (864 Monark). Heart rate was recorded by electrocardiography at rest and then every two minutes before each $30 \mathrm{~W}$ increment in work rate. Maximum heart rates were compared with those recorded in a similar incremental test in eight of the controls. ${ }^{15}$ Maximum heart rate was predicted for each subject with the formula: $210-($ age $\times 0 \cdot 65){ }^{16}$

Maximal voluntary contractions of the quadriceps were measured by dynamometry in 22 patients and all controls, who sat with their hips and knees at $90^{\circ}$. The isometric force exerted at the ankle was recorded as each subject attempted to extend the knee against an inextensible strap placed above the malleoli; it was compared with a normal range related to body weight. ${ }^{13}$ Stimulated twitches of quadriceps were superimposed on the voluntary contraction by placing two large pad electrodes $(14 \times 12 \mathrm{~cm})$ over the anterior thigh and stimulating the muscle at $1 \mathrm{~Hz} .{ }^{17}$ With this technique of interpolating twitches the force is increased by the twitches only when voluntary contractions are submaximal.

Fatigability was studied in the adductor pollicis as the whole muscle can be safely stimulated supramaximally through its motor nerve. Details of the equipment used and experimental procedure have been given elsewhere. ${ }^{14}$ Briefly, before all experiments, the temperature of the muscle was standardised by warming the hand and forearm in a water bath at $45^{\circ} \mathrm{C}$ for 10 minutes; this temperature was maintained throughout the experiment with a lamp. Stimulated twitches $(1 \mathrm{~Hz})$ were used to locate the optimum site and voltage for supramaximal stimulation of the ulnar nerve at the wrist. The computer driven stimulator delivered trains of stimuli in a set pattern of frequencies of $1,10,20,50,100$, and $1 \mathrm{~Hz}$ for one second each (except that $10 \mathrm{~Hz}$ was given for two seconds). The 
isometric forces that resulted were measured by a strain gauge connected to the proximal phalanx of the thumb by an inextensible band. The force signal was also differentiated with respect to time and was used to calculate the maximum relaxation rate (that is, the maximum proportion of force lost in $10 \mathrm{~ms}$ ) after stimulation at $100 \mathrm{~Hz} \cdot{ }^{18}$ Surface electromyography over the adductor pollicis was used to measure excitation (the amplitude of compound muscle action potential). Simultaneous oscillographic recordings of force, force differential (relaxation rate), and excitation were termed the programmed stimulation electromyogram. ${ }^{1419}$

Contractile properties were examined during the fatiguing activity with and without arterial occlusion. Nine patients and nine controls underwent fatiguing activity with arterial occlusion. A baseline programmed stimulation electromyogram was obtained with unfatigued muscle. A sphygmomanometer cuff was inflated around the upper arm and maintained at 100 $\mathrm{mm} \mathrm{Hg}$ above systolic blood pressure. Fatiguing activity consisted of 15 repetitions of the stimulus pattern with a five second rest between each pattern. Aerobic recovery was monitored by repeating the pattern $0 \cdot 5,1,2,3,5,10$, and 15 minutes after the end of activity.

In addition, six patients and nine controls underwent fatiguing activity without arterial occlusion to assess the possible contribution of circulatory abnormalities to altered muscle function. After a baseline programmed stimulation electromyogram had been obtained the fatiguing activity was started. It consisted of 50 patterns of stimuli repeated as above. Recovery was monitored as above. Studies were performed in random order and at least one week apart in subjects who underwent both protocols to ensure full recovery of the adductor pollicis.

The force at $20 \mathrm{~Hz}$ divided by the force at $50 \mathrm{~Hz}$ (the $20: 50 \mathrm{~Hz}$ ratio) and the maximum tetanic force during the $100 \mathrm{~Hz}$ stimulation (in Newtons) were calculated from each baseline electromyogram. The force and excitation at each frequency during muscle activity and the maximal relaxation rate after $100 \mathrm{~Hz}$ stimulation were expressed as percentages of the equivalent values in the baseline electromyogram. Measurements were taken from each electromyogram during activity with occlusion and from every fifth electromyogram during activity without occlusion. Curves expressing force at each frequency as a percentage of that at $100 \mathrm{~Hz}$ in unfatigued muscle were plotted. ${ }^{12}$ Comparisons between patients and normal subjects were made with the two sample $t$ test.

\section{Results}

In the exercise test with the cycle ergometer the maximum heart rate predicted for the controls was 190 (SD 2) beats/min and that attained was 190 (14) beats/ min - that is, $100(7) \%$ of the predicted rate. In the patients the predicted maximum heart rate was $186(5)$ beats/min but the rate reached at exhaustion was only $166(22)$ beats $/ \mathrm{min}$ - that is, $89(12) \%$ of the predicted rate.

Figure 1 shows the maximal voluntary contractions of quadriceps in relation to body weight for the 22 patients. Voluntary efforts did not cause pain in any participant. All patients who generated forces at or below the lower end of the normal range showed increases in force when twitches were interpolated. Results for the controls were within the normal range given by Edwards et al. ${ }^{13}$

The programmed stimulation electromyogram for unfatigued muscle in the patients was similar to that in normal subjects ${ }^{14}$ and the curves relating frequency and force were identical (fig 2). The mean maximum

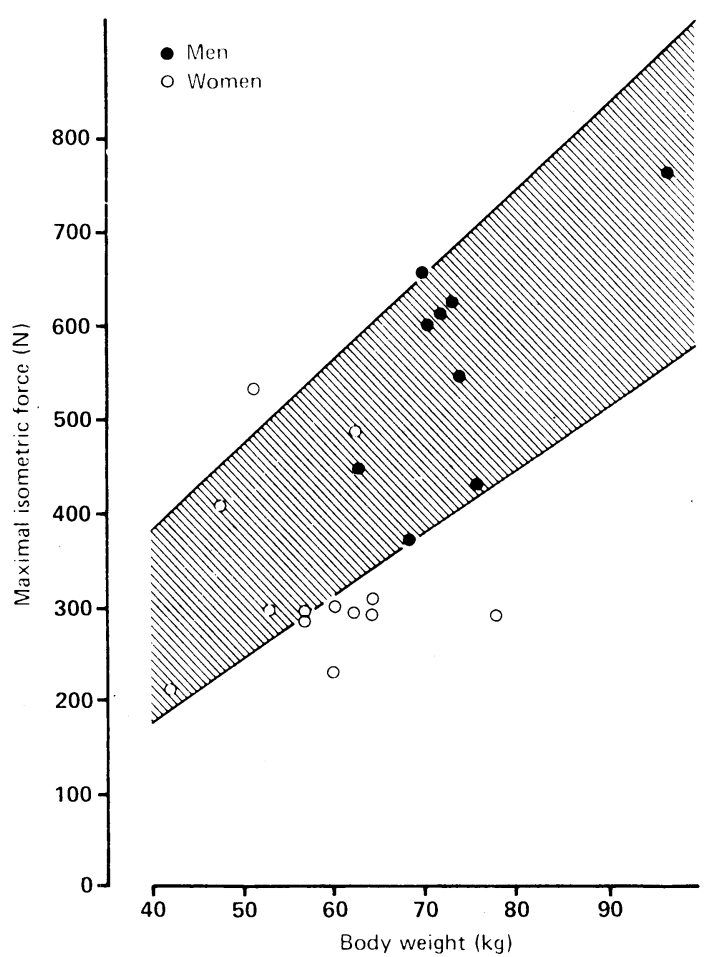

FIG 1 -Strength of quadriceps related to body weight in patients with effort syndromes. Shaded area is range in normal controls ${ }^{13}$

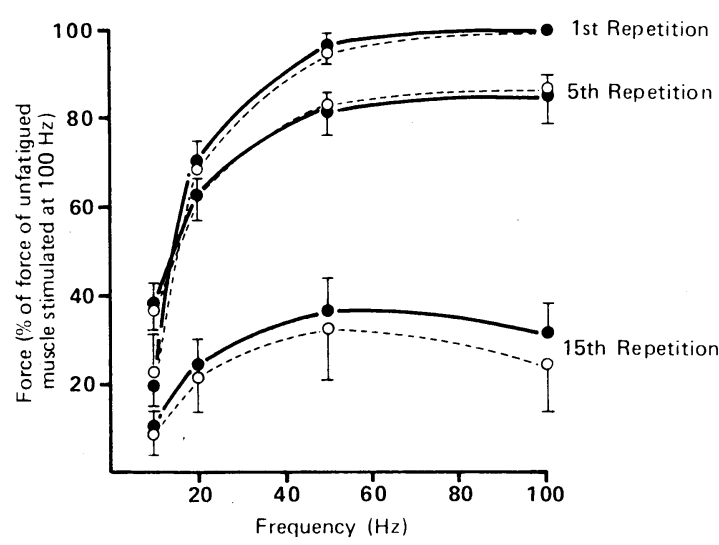

FIG 2-Frequency of stimulation $(\mathrm{Hz})$ related to mean force of contraction (as \% of force of unfatigued muscle) in adductor pollicis during fatiguing contractions with occlusion of circulation in patients with effort syndromes $(\mathbf{O})$ and controls $(\bigcirc)$. Bars indicate ISD

tetanic forces produced in 20 of the patients and the 20 controls were $68(22)$ and $71(18) \mathrm{N}$, respectively; the mean $20: 50 \mathrm{~Hz}$ ratios were $0.71(0.04)$ and $0.72(0.04)$; and the maximal relaxation rates were $11.3(0.9)$ and $11 \cdot 7(0.7) \%$ in $10 \mathrm{~ms}$. None of these results differed significantly between the groups.

Changes in force during occluded activity depended on the frequency of stimulation as previously described by Cooper $e a^{1 / 4}$ and were similar in the two groups (fig 2). Decreases in excitation also depended on frequency and were similar in both groups (table). At the end of activity the maximal relaxation rate fell to $17(5) \%$ of that in unfatigued muscle in the patients and to $16(5) \%$ of that in the controls. After 15 minutes of recovery the $20: 50 \mathrm{~Hz}$ ratio was $0.57(0.05)$ in the patients and $0.53(0.06)$ in the controls, indicating fatigue at low frequencies of stimulation..$^{20}$ Neither of these differences was significant.

The force of contraction and amplitude of excitation fell similarly in the patients and controls during activity without occlusion (table). The changes in the curves relating frequency and force were similar to those during occluded activity but were less pronounced. The maximal relaxation rate fell to $55(12) \%$ 
Mean (SD) amplitude of excitation (as percentage of amplitude produced in unfatigued muscle) during last repetition of pattern of stimulated activity of adductor pollicis

\begin{tabular}{ccc}
\hline Frequency & Patients & Controls† \\
\hline & Arterial occlusion $(15$ repetitions of pattern) \\
$1 \mathrm{~Hz}$ & $86(10)$ & $77(11)$ \\
$10 \mathrm{~Hz}$ & $77(14)$ & $68(17)$ \\
$20 \mathrm{~Hz}$ & $61(18)$ & $52(20)$ \\
$50 \mathrm{~Hz}$ & $31(9)$ & $25(12)$ \\
$100 \mathrm{~Hz}$ & $25(11)$ & $22(11)$ \\
$1 \mathrm{~Hz}$ & $71(6)$ & $72(6)$ \\
& & \\
$1 \mathrm{~Hz}$ & No arterial occlusion (50 repetitions of pattern) \\
$10 \mathrm{~Hz}$ & $87(15)$ & $81(22)$ \\
$20 \mathrm{~Hz}$ & $98(8)$ & $90(18)$ \\
$50 \mathrm{~Hz}$ & $89(9)$ & $91(11)$ \\
$100 \mathrm{~Hz}$ & $68(13)$ & $68(19)$ \\
$1 \mathrm{~Hz}$ & $38(9)$ & $42(9)$ \\
& $76(14)$ & $73(19)$
\end{tabular}

None of the differences between groups was significant.

* Measurements made during arterial occlusion in nine patients and without

† Measurements made in nine controls.

in the patients and to $54(5) \%$ in the controls. After 15 minutes' recovery the $20: 50 \mathrm{~Hz}$ ratio was $0.50(0 \cdot 1)$ in patients and $0.50(0.06)$ in the controls. Neither of these differences was significant.

\section{Discussion}

Our results clearly indicate that contractile properties of skeletal muscle and recovery of muscle after activity are normal in patients with effort syndromes. Abnormalities of performance occur only during voluntary activity, thereby indicating impairment of central mechanisms.

In normal subjects maximal force may be achieved both by voluntary activity and with supramaximal stimulation of the motor nerve. ${ }^{12}$ Our finding that the stimulated strength of the adductor pollicis in patients was similar to that in normal subjects therefore implies a normal strength on voluntary movement. In patients who showed weakness of the quadriceps the positive response to interpolation of twitches pointed to submaximal effort rather than intrinsic weakness. These "weak" patients also showed considerable variability on repeated testing compared with patients with normal strength. Failure to produce maximal voluntary force can be due to a lack of motivation or to pain or fear of pain..$^{21}$ Reduced activation may be due to reflex inhibition by afferent activity arising from damaged painless joints. ${ }^{22}$ None of the patients in our study complained of pain during isometric testing or had clinical evidence of joint disease. Nevertheless, afferent activity arising from muscle itself may also cause reflex inhibition during activity. ${ }^{23}$ As immunological abnormalities have been observed in some patients with postviral fatigue a virus or an inflammatory reaction in the muscle might give rise to afferent stimuli that produce alterations in motor neurone drive $^{824}$; the persistence of symptoms may thus be a learnt response to a stimulus that is no longer present.

This study failed to show electromyographic changes, although previous findings suggested an abnormality of the sarcolemmal membrane. ${ }^{9}$ Acute viral infections can affect neuromuscular transmission with reduced voluntary strength and endurance, but a full recovery is expected. . $^{25}-27$

Subjects who exercised on a cycle ergometer stopped the exercise when the effort was no longer bearable. The patients who did not reach their predicted maximum heart rates were those who also showed central weakness on testing of the quadriceps on voluntary movement, which suggests that the reduced maximal capacity may be due to reduced tolerance of effort symptoms.

Reduced generation of force at low frequency, which persists for some hours after exercise in normal muscle (low frequency fatigue ${ }^{20}$ ), is associated with increased activity of motor neurones for a given force ${ }^{28} 29$ and with increased perception of effort. ${ }^{30}$ Whether the increased perception is the cause or result of the increased motor neurone activity is not known. In our study the $20: 50 \mathrm{~Hz}$ ratios and curves relating frequency and force for the patients with effort syndromes were identical with those for the normal subjects, ruling out an excess of low frequency fatigue as a cause of the patients' symptoms.

Great caution is needed in interpreting the results of this study and drawing conclusions about treatment as the findings clearly point to lack of central drive or motivation. It is all too easy to adopt an unsympathetic attitude, which is unhelpful to the patient, who may be offended and subsequently become suspicious of any physician or psychiatrist. Medical practitioners must remain open minded about the causes of effort syndromes; for instance, this study does not exclude the possibility of afferent activity from muscle impairing voluntary performance.

Even if a virus is detected in serum and perhaps in muscle could the results of this study be used to help treat patients, and what advice should they be given? Patients are often told simply to rest, but resting may aggravate the symptoms by causing cardiovascular unfitness. The patients with effort syndromes in this study had muscle that responded normally to exercise even though they experienced fatigue prematurely. This evidence can be used to reassure patients that it is safe to exercise, even though exercising may make them feel worse. Anxiety about tachycardia and breathlessness can therefore be allayed, and the vicious cycle of unfitness and fatigue ${ }^{4}$ can be broken by carefully and gradually increasing the amount of activity.

For patients who either do not respond to or are unwilling to try this approach to treatment further consideration of psychological influences may be necessary. Psychological factors often complicate effort syndromes, particularly if the patient has suffered from lack of understanding from family, friends, and medical practitioners. Such psychological problems should be identified by careful assessment and treated. Whatever the causes of effort syndromes, a coping strategy is required while there is no specific treatment. Behavioural therapy using the principles for managing chronic pain may prove useful for increasing tolerance to exercise. An integrated approach giving advice on exercise as well as psychological support ${ }^{10}$ seems most helpful in returning these patients to normal functioning.

We thank the participants in this study and are grateful for support from the Muscular Dystrophy Group of Great Britain and Northern Ireland and from ICI Pharmaceuticals (UK).

1 Lewis T, ed. The "effort syndrome," its signs and symptoms, contrasted with those with heart disease. In: Soldier's heart and effort syndrome. London: Shaw and Sons, 1918:10-26.

2 Wood P. Aetiology of Da Costa's syndrome. Br Med f 1941;i:845-51.

3 Newham D, Edwards RHT. Effort syndrome. Physiotherapy 1979;65:52-6.

4 Edwards RHT. Muscle fatigue and pain. Acta Med Scand 1986;711(suppl): 179-88.

5 Behan PO, Behan WMH, Bell EJ. The post-viral fatigue syndrome: an analysis of the findings in 50 cases. $\mathcal{F}$ Infect 1985;10:211-22.

6 Anonymous. Epidemic myalgic encephalomyelitis [Editorial]. $\mathrm{Br}$ Med $\mathcal{J}$ 1978;i:1436-7.

7 Ramsay AM, ed. Events of the momentous year 1955. In: Postviral fatigue syndrome: the saga of Royal Free disease. London: Gower Medical, 1986:1-11.

8 Yousef GE, Mann GF, Smith DG, et al. Chronic enterovirus infection in patients with postviral fatigue syndrome. Lancet 1988;i:146-50.

$9 \mathrm{Jamal}$ GA, Hansen S. Electrophysiological studies in the postviral fatigue syndrome. I Neurol Neurosurg Psychiatry 1985;48:691-4.

10 David AS, Wessely S, Pelosi AJ. Postviral fatigue syndrome: time for a new approach. Br Med f 1988;296:696-9.

11 Edwards RHT. Human muscle function and fatigue. In: Porter R, Whelan J, eds. Human muscle fatigue: physiological mechanisms. London: Pitman Medical, 1981:1-18. (Ciba Foundation symposium No 82.)

12 Merton PA. Voluntary strength and fatigue. F Physiol 1954;123:553-64.

13 Edwards RHT, Young A, Hosking GP, et al. Human skeletal muscle function: description of tests and normal values. Clin Sci Mol Med 1977;52:283-90. 
14 Cooper RG, Edwards RHT, Gibson H, Stokes M. Human muscle fatigue: frequency dependence of excitation and force generation. $f$ Physiol 1988;397:585-99.

15 Cooper RG, Stokes MJ, Edwards RHT, Stark RD. Absence of excess peripheral muscle fatigue during $\beta$ adrenoceptor blockade. Br f Clin Pharm 1988:25:405-16.

16 Astrand I. Aerobic work capacity in men and women with special reference to age. Acta Physiol Scand 1960;169(suppl):45-60.

17 Chapman SJ, Edwards RHT, Greig C, Rutherford O. Practical application of the twitch interpolation technique for the study of voluntary contraction of the quadriceps muscle in man. $\mathscr{7}$ Physiol 1984;353:3P.

18 Wiles CM, Young A, Jones DA, Edwards RHT. Relaxation rate of constituent muscle fibre types in human quadriceps. Clin Sci 1979;56:47-57.
mus

9 Edwards RHT. Physiological analysis of skeletal muscle weakness and fatigue. Clin Sci Mol Med 1978;54:463-70.

20 Edwards RHT, Hill DK, Jones DA, Merton PA. Fatigue of long duration in human skeletal muscle after exercise. $\mathcal{F}$ Physiol 1977;272:769-78.

21 Basmajian JV. Re-education of vastus medialis; a misconception. Arch Phys Med Rehabil 1970;51:245-7.

22 Stokes M, Young A. The contribution of reflex inhibition to arthrogenous muscle weakness. Clin Sci 1984;67:7-14.
23 Woods JJ, Furbush F, Bigland-Ritchie B. Evidence for a fatigue-induced reflex inhibition of motoneuron firing rates. F Neurophysiol 1987:58:125-37. 24 Ho-Yeu DO, Carrington D, Armstrong AA. Myalgic encephalomyelitis and alpha-interferon. Lancet 1988;i:125.

25 Friman G, Schiller HH, Schwartz MS. Disturbed neuromuscular transmission in viral infections. Scand f Infect Dis 1977;9:99-103.

26 Friman G. Effect of acute infectious disease on isometric muscle strength. Scand f Clin Lab Invest 1977;37:303-8.

27 Friman G. Effect of acute infectious disease on human isometric muscle endurance. Upsala J Med Sci 1978;83:105-8.

28 Edwards RG, Lippold OCJ. The relation between force and integrated electrical activity in fatigued muscle after exercise. $\mathcal{F}$ Physiol 1956;132: 677-8

29 Komi PV, Viitalaso JT. Changes in motor unit activity and metabolism in human skeletal muscle during and after repeated eccentric and concentric contractions. Acta Physiol Scand 1977;100:246-54.

30 Gandevia SC, McCloskey DI. Interpretation of perceived motor commands by reference to afferent signals. $\mathcal{F}$ Physiol 1978;283:493-9.

31 Savage RA, Stokes MJ, Reilly T, Edwards RHT. Increased perception of effort with muscle fatigue. Int $\mathcal{f}$ Rehab Res (in press).

(Accepted 19 Julv 1988)

\title{
Prevention of gastroduodenal damage induced by non-steroidal anti-inflammatory drugs: controlled trial of ranitidine
}

\author{
R S B Ehsanullah, M C Page, G Tildesley, J R Wood
}

\section{Abstract}

Objective-To evaluate the prophylactic effect of ranitidine $150 \mathrm{mg}$ twice daily in patients requiring one of the following non-steroidal anti-inflammatory drugs: naproxen, piroxicam, diclofenac, and indomethacin. In addition, risk factors were studied in order to help in targeting of such treatment to specific groups of patients.

Design-Double blind, placebo controlled, randomised, parallel group with endoscopic assessments at 0,4 , and 8 weeks.

Setting-Multicentre outpatient study at secondary referral centres in five European countries.

Patients -297 patients with rheumatoid arthritis or osteoarthritis over the age of 18 without lesions in the stomach and duodenum at baseline endoscopy (after one week without taking non-steroidal antiinflammatory drugs). Those taking other antirheumatic agents, concomitant ulcerogenic drugs, or treatment for peptic ulcers within the previous 30 days were excluded. Age, sex, arthritic disease, and type of non-steroidal anti-inflammatory drug used were comparable in the two treatment groups. In all, 263 patients completed the trial.

Interventions-Ranitidine $150 \mathrm{mg}$ twice daily or placebo (plus the selected non-steroidal antiinflammatory drug) was prescribed within five days after the baseline endoscopy for two consecutive periods of four weeks. Paracetamol was permitted during the study, but not antacids. Patients were withdrawn if the most severe grade of damage (including ulceration) was found at the four week endoscopy or when indicated, or with lesser damage at the investigator's discretion.

End point-Frequency of gastric and duodenal ulceration or lesions, or both.

Measurements and main results - The cumulative incidence of peptic ulceration by eight weeks was $10.3 \%(27 / 263)$; 2 out of $135(1.5 \%)$ developed duodenal ulceration in the ranitidine group, compared with 10 out of $126(8 \%)$ taking placebo. The frequency of gastric ulceration was the same $(6 \%)$ for the two groups at eight weeks. Though significantly fewer gastric lesions developed in the ranitidine group by four weeks, this difference was not evident by eight weeks. The frequency of non-ulcerative lesions in the duodenum did not differ greatly for the two groups at either time point. Twelve out of 75
(16\%) patients taking piroxicam developed peptic ulceration, of whom two thirds had duodenal ulceration. Patients with a history of peptic ulcer were particularly susceptible to recurrent ulceration, against which ranitidine offered some protection.

Conclusions-Ranitidine $150 \mathrm{mg}$ twice daily significantly reduced the incidence of duodenal ulceration but not gastric ulceration when prescribed concomitantly with one of four commonly used non-steroidal anti-inflammatory drugs.

\section{Introduction}

Non-steroidal anti-inflammatory drugs for the treatment of arthritis are the most widely prescribed group of drugs world wide. In the United Kingdom 23 million prescriptions were issued in 1986 for this group of drugs, accounting for around 5\% of all NHS prescriptions. These drugs, however, account for $25 \%$ of all the suspected adverse drug reactions reported to the United Kingdom Committee on Safety of Medicines each year and 21\% reported to the United States Food and Drug Administration. ${ }^{12}$ The commonest and most serious of these reactions are gastrointestinal..$^{3-5} \mathrm{Up}$ to $60 \%$ of patients taking these drugs report dyspepsia, ${ }^{5-7}$ but this has not proved to be a reliable guide to the presence of gastroduodenal lesions. ${ }^{8-12}$

Aspirin causes gastroduodenal damage in more than $80 \%$ of subjects, ${ }^{13} 14$ varying from acute microscopic gastric changes ${ }^{15}$ to potentially more serious chronic gastric ulceration or haemorrhage..$^{76-18}$ In a large prospective study gastric lesions were found in a third of patients taking non-steroidal anti-inflammatory drugs for a year and in half of those receiving two or more such drugs. ${ }^{8}$ Langman calculated a rate of one episode of gastrointestinal haemorrhage per 6000 prescriptions for non-steroidal anti-inflammatory drugs $^{19}$; estimates for attributable mortality in the United Kingdom range from $200^{20}$ to over $4000^{21}$ deaths annually. Epidemiological data also point to rising rates of ulcer perforation in the elderly, partly attributable to the increased use of this group of drugs. ${ }^{22}$ Other centres in the United Kingdom have noted this association, ${ }^{23-25}$ though some studies from Australia and America do not show similar trends. ${ }^{26} 27$

Attempts to decrease the risks associated with antiinflammatory treatment by using enteric coated formulations, suppositories, or prodrugs have not 